\title{
Structural Design of a Novel Wearable Knee Exoskeleton
}

\author{
Tang Xiaolu ${ }^{1,}$, Chen Lumin ${ }^{2, b}$ \\ ${ }^{1}$ School of Mechanical \& Electrical Engineering, Zhengzhou University of Light Industry, \\ Zhengzhou, Henan Province, P.R. of China, 450002 \\ ${ }^{2}$ School of Mechanical \& Electrical Engineering, Zhengzhou University of Light Industry, \\ Zhengzhou, Henan Province, P.R. of China, 450002 \\ a1152616635@qq.com, bluminchen@zzuli.edu.cn
}

Keywords: Human - machine compatibility, Knee rehabilitation, Exoskeleton, Self-aligning

Abstract. Based on the structure characteristics of the human physiological knee joints during the flexion and extension movements, we design a self-aligning wearable knee exoskeleton mechanism considering the principle of human - machine compatibility. The mechanism's mathematical model is established using the D-H notation, then the kinematic analysis is carried out by MATLAB and the workspace of the mechanism is calculated. The computational results show that the workspace of the human knee joint's microscopic mechanism during the flexion and extension movements is contained within the that of self-aligning knee joint exoskeleton. Therefore the research will provide a novel type of wearable knee exoskeleton with fewer side effects for medical rehabilitation patients.

\section{Introduction}

Population aging has become an inevitable trend of social development, which is more prominent for patients with stroke. In addition, due to traffic accidents, sports injuries and other acquired factors that lead to impairment of motor ability is also growing. The rehabilitation of lower limbs has become a problem that must be faced by society. As a result, many scientific researchers are conducting in developing rehabilitative exoskeletons of lower limbs.

In the human knee joint, the femur and tibia both rotates and translates while the knee is bent. As a result, the rotation center moves with respect to the bending angle of the human knee. Consequently, the rotation centers of knee joints in the human body and exoskeleton become inconsistent with bending of the knees[1]. Misalignment of the joint axes of rotation may cause additional load on the wearer's joints[2].Therefore, the study of the knee exoskeleton with compliant joints has become a necessity in the development of the lower-limb exoskeleton.

There are three types of the existing knee joint exoskeleton: First, the knee-joins model is usually simplified as a pin-joint which has only one-DOF with a fixed rotation axis. Now, Most of the knee exoskeletons are used in this way. For example, SERKA[3], a representative rehabilitation[4]. Second, in the sagittal plane, the rotating axis of the human physiological knee is not a point, but a closed plane curve that is similar to a shape of "J" [5]. According to the approximate "J" curve, a four-bar linkage mechanism was designed based on the Instantaneous center of rotation (ICOR) [6]. In this way, motion curves of the instantaneous center of rotation for both of human and exoskeleton are similar. Thirdly, the knee-joints are designed as a grooved cam based on the approximate "J" curve. Combined with a grooved cam[7], in this kind of design shank can slide and rotate about the far end of thigh bone when a human knee-joint flexing freely. 
However, the way of knee-joints' sliding and rotating movements depends on the physical structure and shape of the tibia and femur. Since each human's knee joint is "unique"[8], the fixed trajectory curves of four-bar linkage knee-joints mechanism and the grooved cam mechanism can hardly match the different "J" curves of each human. Therefore, existing exoskeleton knee joints may cause additional harms to the wearers.

In this paper we will design a self-aligning knee flexible exoskeleton, with simple structure and comfortable wearing property. The trajectory of the instantaneous center of rotation of this design is a limited sized plane region, and all possible "J" curves are put inside the boundary of it. This design increases the compatibility between the human and the exoskeleton, reducing the damage caused by the additional load on the human knee joints.

\section{Structural design}

\section{Analysis of the flexion and extension movements' structure of human knee joint}

According to the analysis of human knee joint structure, Walker et al, the joint's instantaneous center is a momentary helical axis in three-dimensional space, and the motion form is complex[9]. It is almost impossible to completely replicate human knee joint motion. Therefore, on the basis of the movement of the knee joint in the sagittal plane, the knee-joints exoskeleton should be adapted to the three-dimensional motion of the human body. As shown in Fig. 1, the instantaneous center of rotation of the leg relative to the thigh during flexion and extension movements of the knee joint is not constant point, but is constantly changing. The trajectory of the instantaneous center of rotation in the sagittal plane is a plane curve that is similar to a shape of "J". It can be seen from the literature when the human knee joint movements of flexion and extension, at different rotation angles, the relative translational distance between the femur and the tibia in the human knee joint is not the same. The femur of the knee joint of the human body begins to move forward about $2 \mathrm{~mm}$ at $10^{\circ}$. The maximum translational value is $14 \mathrm{~mm}$ at $140^{\circ}$ flexion. And in the process of knee flexion and extension, accompanied by pronation/external rotation, adduction/abduction, the maximum deflection angle of $13^{\circ}$ to 7 。[10].

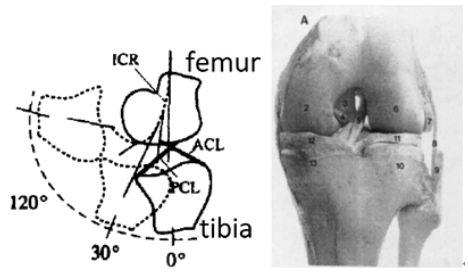

Fig. 1 Trajectory of the instantaneous center of rotation

\section{Geometrical and Physical model}

Lower extremity exoskeleton is worn on the human leg, we must consider the Human - machine compatibility. Using the self-aligning device, the instantaneous center of rotation of both the knee exoskeleton and the human knee joint can be kept coincident. It greatly reduces the additional load that the exoskeleton brings to the human body, reduces the mutual force between the human and robot, and releases the freedom of the human knee-joints. By Solid Works 3D Software to construct the whole model. As shown in Fig. 2, the most important of these is the self-aligning device[11], which adds a redundant, two-degree-of-freedom movement to adjust the knee joint's position of rotating axis[12]. At the same time, the sphere-pin pair[13] between the lower leg bandage and the lower leg connecting frame is a passive kinematic pair, which is adapted to the rotating axis of the human knee joint so as to keep the alignment in the part of the rotating axis between exoskeleton and the human in the three-dimensional space. When the knee-joints exoskeleton help the body to stand support, the shell and the Connection frame of leg play a supporting role in the way of a point 
contact. Meanwhile, the connecting rod 1 in the self-aligning device is pressed on the stopper, which plays a secondary support role.

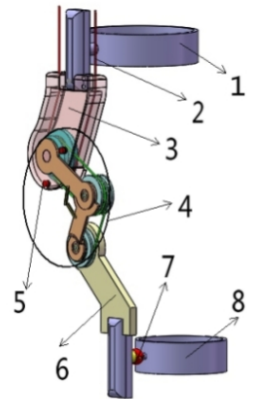

Fig. 2Three-dimensional model of the self-aligning knee-joint exoskeleton device

1. Thigh bandage 2.Driving cable 3.Shell 4.Self-aligning device 5.Stopper 6.Connection frame of leg 7.Sphere-pin pair 8.Leg bandage

As shown in Fig. 3, the principle of the self-aligning device. The driving pulley is fixedly connected with the aligning pulley 1 and can transmit the same torque, the aligning pulley 1 and the aligning pulley 2 are connected by the connecting rod 1, and the aligning pulley 2 and the aligning pulley 3 are connected by the connecting rod 2 . The aligning pulley 1 , the aligning pulley 2 and the aligning pulley 3 pass in order the same torque through the aligning cable. Therefore, the aligning pulley 3 has the same torque as the driving pulley. The aligning pulley 3 is fixedly connected to the lower leg shank and provides torque. The device consists of two parallelogram serial structures, which transfer the torque from the thigh to the leg and the movement of the joints is independent with the position of rotating axis, so that the accurate restriction of the human joint can be relieved. When the knee is flexed, the connecting rod 1 and the connecting rod 2 can be rotated simultaneously to adjust the most important position of rotated axis of the knee-joints exoskeleton in the sagittal plane. The adjustment range is $\Delta \mathrm{dx}$ to $\Delta \mathrm{dy}$.

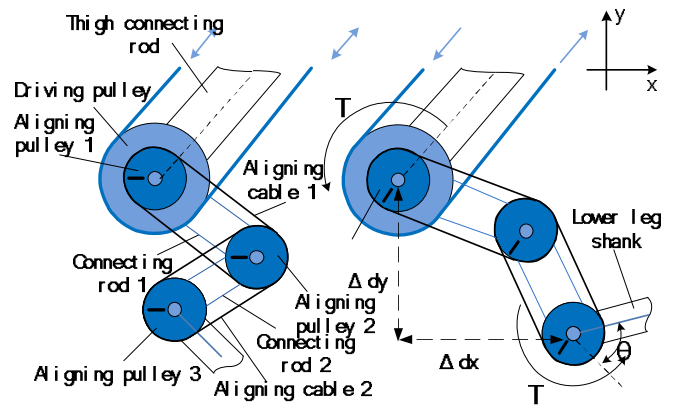

Fig. 3Illustrative diagram of the self-aligning device

\section{Kinematics modeling and simulation}

\section{D-H notation}

In the study of the robot kinematics, the D-H notation is the most commonly used. From the view of structural design, the knee-joints exoskeleton is composed of a series of rigid bodies, and each component is simplified as a connecting rod. The multi-degree of freedom of the knee is decomposed into several single degrees of freedom joint, then analysis of kinematics modeling of the knee exoskeleton is carried out.

In the sagittal plane, the Self-aligning knee joint exoskeleton has three degrees of freedom, one active degree of freedom of rotation, two passive degrees of freedom of translating. As shown in Fig. 4, the mechanism has three rotational pairs and a D - $\mathrm{H}$ notation is set up, in whichO $\mathrm{O}_{1} \mathrm{O}_{2}$ and $\mathrm{O}_{3}$ are the 3 rotational pairs in the knee joint. Assuming that the thigh is fixed, $\mathrm{O}_{0}-\mathrm{X}_{0} \mathrm{Y}_{0} \mathrm{Z}_{0}$ is a 
base coordinate system established between the thigh and the knee joint. The coordinate system of each rotational pair is $\mathrm{O}_{1}-\mathrm{X}_{1} \mathrm{Y}_{1} \mathrm{Z}_{1}, \mathrm{O}_{2}-\mathrm{X}_{2} \mathrm{Y}_{2} \mathrm{Z}_{2}$, and $\mathrm{O}_{3}-\mathrm{Z}_{3} \mathrm{Y}_{3} \mathrm{Z}_{3}$. The interlace angle between the axis $X_{i-1}$ and the axis $X_{i}$ is $\theta_{i}$. That is $\theta_{1}, \theta_{2}$, and $\theta_{3}$. The length of each connecting rod is $1_{1}, l_{2}$. According to the movement of the knee joint exoskeleton, the motion parameters of the change matrix in the D-H notation are shown in Table 1.

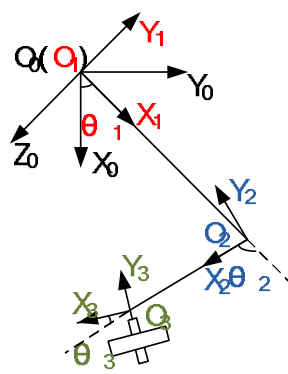

Fig. 4 Diagram of D-H notation

Table 1. Motion parameters of D-H notation

\begin{tabular}{ccccccc}
\hline Renumber & Joint & $\alpha_{\mathrm{i}-1}$ & $\mathrm{a}_{\mathrm{i}-1}$ & $\mathrm{~d}_{\mathrm{i}}$ & $\cos \alpha_{\mathrm{i}-1}$ & $\sin \alpha_{\mathrm{i}-1}$ \\
\hline 1 & $\theta_{1}$ & 0 & 0 & 0 & 1 & 0 \\
2 & $\theta_{2}$ & 0 & $1_{1}$ & 0 & 1 & 0 \\
3 & $\theta_{3}$ & 0 & $\mathrm{l}_{2}$ & 0 & 1 & 0 \\
\hline
\end{tabular}

According to table 1 , we can get the changes in the coordinates of each rotation center, seeing matrix 1, matrix 2, matrix 3; The spatial position of rotating axis and rotating angle that is relative to the base coordinate system can be calculated in the sagittal plane.

$$
\begin{array}{ll}
\mathrm{T}_{1}^{0}=\left[\begin{array}{cccc}
\cos \theta_{1} & -\sin \theta_{1} & 0 & 0 \\
\sin \theta_{1} & \cos \theta_{1} & 0 & 0 \\
0 & 0 & 1 & 0 \\
0 & 0 & 0 & 1
\end{array}\right](1) & \mathrm{T}_{2}^{1}=\left[\begin{array}{cccc}
\cos \theta_{2} & -\sin \theta_{2} & 0 & 1_{1} \\
\sin \theta_{2} & \cos \theta_{2} & 0 & 0 \\
0 & 0 & 1 & 0 \\
0 & 0 & 0 & 1
\end{array}\right] \text { (2) } \\
\mathrm{T}_{3}^{2}=\left[\begin{array}{cccc}
\cos \theta_{3} & -\sin \theta_{3} & 0 & 1_{2} \\
\sin \theta_{3} & \cos \theta_{3} & 0 & 0 \\
0 & 0 & 1 & 0 \\
0 & 0 & 0 & 1
\end{array}\right](3) & \mathrm{T}_{3}^{0}=\left[\begin{array}{cccc}
\mathrm{C}_{123} & -\mathrm{S}_{123} & 0 & 1_{1} \mathrm{c}_{1}+l_{2} \mathrm{c}_{2} \\
\mathrm{~S}_{123} & \mathrm{C}_{123} & 0 & 1_{1} \mathrm{~s}_{1}+1_{2} \mathrm{~s}_{2} \\
0 & 0 & 1 & 0 \\
0 & 0 & 0 & 1
\end{array}\right](4)
\end{array}
$$

In matrix $4, \mathrm{C}_{123}=\cos \left(\theta_{1}+\theta_{2}+\theta_{3}\right), \mathrm{S}_{123}=\sin \left(\theta_{1}+\theta_{2}+\theta_{3}\right), \mathrm{C}_{12}=\cos \left(\theta_{1}+\theta_{2}\right), \mathrm{C}_{1}=\cos \theta_{1}, \mathrm{~S}_{1}=\sin \theta_{1}$; $\theta_{1} \in\left[\frac{\Pi}{6}, \frac{4 \Pi}{9}\right], \theta_{2} \in\left[\frac{-7 \Pi}{18}, \frac{-13 \Pi}{18}\right], 1_{1}=90 \mathrm{~mm}, 1_{2}=75 \mathrm{~mm}$.

The parameter $\theta_{1}, \theta_{2}, \theta_{3}, l_{1}$ and $l_{2}$ is substituted into the knee joint exoskeleton motion matrix 4 . Through MATLAB programming and simulation, we can get the range of the change of the active rotation joint axis of the knee joint in the sagittal plane. As shown in Fig. 5, the approximate shape is a quadrilateral, the adjustment range is $\Delta \mathrm{dx} \epsilon[38 \mathrm{~mm}, 86 \mathrm{~mm}]$ and $\Delta \mathrm{dy} \epsilon[-18 \mathrm{~mm}, 63 \mathrm{~mm}]$. It was previously mentioned that each human knee joint is "unique", the approximate range of the "J" curve is $14 \mathrm{~mm}$ in length and $8 \mathrm{~mm}$ in height. The different "J" curve of each human knee is slightly adjusted in the sagittal plane, but all of the "J" curves are included in the adjustment range. In summary, due to the range of the position of active rotating axis of the exoskeleton in the sagittal plane includes the change range of that of the human knee-joints. So, within the range of $\Delta \mathrm{dx}$ and 
$\Delta \mathrm{dy}$, with the flexion and extension movements, the position of rotating axis of the knee-joints exoskeleton can passively align with a human knee-joint flexing freely.

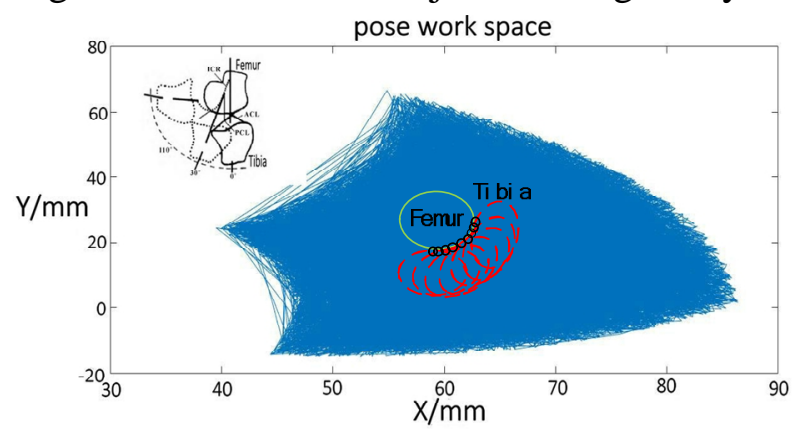

Fig. 5 Working space of the rotating axis of the knee-joints exoskeleton (Sagittal plane)

\section{Conclusions}

According to the human - machine compatibility, considering the comfortable and safe requirements, a novel self-aligning knee flexible exoskeleton, the rotational center of which is a plane with a larger envelope than that of human's physiological knee joints, is designed. This design allows the exoskeleton and the human body automatically keep alignment in the knee-joints axis of rotation during the movement of the three-dimensional space, which will greatly reduce the additional load on the human body.

\section{References}

[1]Wang Jianping. Human knee joint tibiofemoral joint motion characteristics analysis in China[J]. Journal of clinical anatomy, 2017,35 (01): 62-68.

[2]Zhang Jiafan. Flexible exoskeleton human-machine intelligent system in china[M]. Beijing: Science Press, 2011:66 - 67

[3] Sulzer J S, Roiz R A, Peshkin M A, et al. A highly back drivable, lightweight knee actuator for investigating gait in stroke[J]. IEEE Transactions on Robotics, 2009, 25(3):539-548.

[4]DOLLAR A M, HERR H. Lower extremity exoskeletons and active orthoses: challenges and state-of-the-art[J].IEEE transactions on robotics, 2008, 24(01) : 144-158.

[5]Yang Zhuanglai. Human structurology in china[M]. Beijing: Higher Education Press, 2004:51-87

[6]Dong Jin Hyun. Biomechanical design of an agile, electricity-powered lower-limb exoskeleton for weight-bearing assistance [J]. Robotics \& Autonomous Systems, 2017, 95.

[7]WANG Donghai, LEE K M , GUO Jiajie, et al. Adaptive knee joint exoskeleton based on

biological geometries[J] . IEEE / ASME transactions on mechatronics, 2014, 19 (04) :1268-1278 .

[8]Wang Jianping. Analysis of relative motion of human knee patellar joint [J]. Journal of Shanghai Jiao Tong University, 2009, 43 (7): 1043-1046.

[9] S.Walker, H.Kurosawa,S.Rovick.Externalkneejointdesignbasedonnormalmotion[J]. Journal of Rehabilltation Research and Development, 1985, 22(1): 9 - 22.

[10] Wang J, Tao K, et al. Modelling and analysis on biomechanical dynamic characteristics of knee flexion kinematics under squating [J].Scientific World Journal, 2014,(2014): 321080-321080.

[11] Lee Y, Kim Y J, Lee J, et al. Biomechanical Design of a Novel Flexible Exoskeleton for Lower Extremities[J]. IEEE/ASME Transactions on Mechatronics, 2017, PP(99):1-1.

[12] Celebi B, Yalcin M, Patoglu V. Assist On-Knee: A self-aligning knee exoskeleton[C]// Ieee/rsj 
International Conference on Intelligent Robots and Systems. IEEE, 2013:996-1002.

[13]Li Jianfeng. Synthesis and structural design of the conformation of knee joint exoskeleton in china[J]. Journal of Harbin Engineering University, 2017,38 (04): 625-632. 